\title{
Rezension: Andrea Egbuna-Joss, Der Schutz der öffentlichen Ordnung und Sicherheit im Rahmen der internationalen Schutzgewährung
}

Stefan Schlegel *

Die Dissertation von Andrea Egbuna-Joss untersucht die die Bestimmungen zum Schutz der öffentlichen Ordnung in der Qualifikationsrichtlinie 2011/95 der EU auf ihre Vereinbarkeit mit völkerrechtlichen Vorgaben. Die Qualifikationsrichtlinie wird zu diesem Zweck in den Kontext anderer regionaler Instrumente und in eine historische Übersicht über die Entwicklung des Flüchtlingsschutzes eingebettet. Der Wert des Beitrags von Egbuna-Joss ergibt sich nicht nur aus der detaillierten und systematischen Besprechung der einschlägigen Bestimmungen der Richtlinie, sondern besonders auch aus der ausgezeichneten und zugänglichen Einführung in deren historischen und rechtsvergleichenden Kontext.

I. Problemstellung. .223

II. Hauptbefund: Kategorienfehler 223

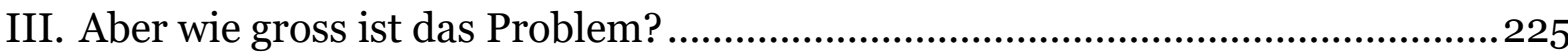

IV. Wie konnte das passieren? ..................................................................226

V. Zum Begriff der Sicherheit und Ordnung .227

Zitiervorschlag: Stefan Schlegel, Rezension: Andrea Egbuna-Joss, Der Schutz der öffentlichen Ordnung und Sicherheit im Rahmen der internationalen Schutzgewährung, in: sui-generis 2017, S. 222

URL: $\quad$ sui-generis.ch/45

DOI: $\quad$ https://doi.org/10.21257/sg.45

* Dr. iur. Stefan Schlegel, Max-Planck-Institut zur Erforschung multiethnischer und multireligiöser Gesellschaften, Göttingen. 


\section{Problemstellung}

1 «Die Qualifikationsrichtlinie 2011/95 sieht vor, dass nicht alle Personen, die internationalen Schutzes bedürfen, auch als solche anerkannt werden sollen (...).» Dieser Satz aus der Dissertation von Andrea Egbuna-Joss (S. 160) bringt den Untersuchungsgegenstand auf den Punkt. Wann dürfen Personen, deren Schutzbedürftigkeit anerkannt ist, aus Gründen der öffentlichen Ordnung und Sicherheit aus dem Status ausgeschlossen werden, den die Anerkennung der Schutzbedürftigkeit an sich nach sich ziehen würde? Egbuna-Joss untersucht diese Frage für die Qualifikationsrichtlinie der EU (RL 2011/95), unter besonderer Berücksichtigung der völkerrechtlichen Vorgaben.

Um den nötigen Hintergrund für die völkerrechtliche Beurteilung der Richtlinie zu schaffen, stellt sie der eigentlichen Analyse zwei Teile voran, einen, in dem sie das Spannungsfeld zwischen Schutzgewährung und den Sicherheitsbedenken der Aufnahmestaaten in seiner historischen Entwicklung und in seiner regionalen Ausprägung darstellt und vergleicht.

3 Ein zweiter Teil stellt das Gemeinsame Europäische Asylsystem in seiner historischen Entwicklung und in seiner institutionellen Ausgestaltung dar. Der dritte Teil widmet sich dann der eigentlichen Kernaufgabe der Untersuchung, dem Schutz der Sicherheit und Ordnung im Rahmen der Qualifikationsrichtlinie.

Während dieser Hauptteil nicht nur als Kapitel einer Dissertation gut funktioniert, sondern auch als sorgfältiger und detaillierter Kommentar zur Systematik und zu den einschlägigen Bestimmungen der Qualifikationsrichtlinie (insbes. Art. 12, Art. 14, Art. 17, Art. 19 und Art. 21-25 RL 2011/95), sind die beiden ersten Teile als eine ausgezeichnete und sehr zugängliche Einführung in die Geschichte des Flüchtlingsschutzes mit den Mitteln des internationalen Rechts seit dem Zweiten Weltkrieg. Besonders wertvoll sind sie als Einführung und Nachschlagwerk für die verschiedenen regionalen Schutzinstrumente ${ }^{1}$ und für den Nachvollzug der Entstehung eines gemeinsamen europäischen Asylsystems.

5 Ein Anhang gibt einen hilfreichen Überblick über alle Artikel in der Qualifikationsrichtlinie, die sich mit dem Schutz der öffentlichen Ordnung und Sicherheit befassen, den Beweisanforderungen, die diese stellen, und den Rechtsfolgen, die sie zeitigen.

\section{Hauptbefund: Kategorienfehler}

6 Zur Untersuchung der Völkerrechtskonformität der Qualifikationsrichtlinie werden deren einschlägige Bestimmungen in drei Kategorien eingeteilt. Bestimmungen über die Nichtgewährung (trotz Schutzbedürftigkeit) des Flüchtlingsoder des subsidiären Schutzstatus für gewisse Personen, Bestimmungen über die Einschränkung der Statusrechte und

\footnotetext{
1 Es erscheint wünschenswert, künftig in Vergleiche von regionalen Mechanismen betreffend den Flüchtlingsschutz noch eine weitere Region mit einzubeziehen, nämlich die Mitgliedstaaten der Shanghai Cooperation Association. In dieser internationalen Organisation ist Flüchtlingspolitik dem Ziel der (Staats-)Sicherheit fast vollständig untergeordnet und die Mitgliedsstaaten sichern sich gegenseitig $\mathrm{zu}$, niemandem einen Flüchtlingsstatus zu gewähren, der in einem anderen Mitgliedstaat wegen «Terrorismus», «Extremismus» oder «Separatismus» verfolgt wird. Vgl. dazu nunmehr David Suter, «The Shanghai Cooperation Organization», Schulthess, 2015, S. 523.
} 
Bestimmungen über die Beendigung der internationalen Schutzgewährung aus Gründen der Sicherheit und Ordnung.

7 Hauptbefund der Untersuchung ist, dass die Richtlinie Kategorienfehler begehe und die aus der historischen Entwicklung des Flüchtlingsschutzes gut etablierten Unterschiede zwischen einem Ausschluss aus der Schutzbedürftigkeit und dem Ausschluss aus einem flüchtlingsrechtlichen Status miteinander vermische. Was bisher strikte voneinander getrennt gewesen sei - der «völkerrechtliche Flüchtlingsschutz und der menschenrechtliche Ausweisungsschutz» - werde durch die Systematik der Richtlinie zusammengeworfen (S. 219). Dieser Befund bezieht sich zunächst sowohl auf die Zuerkennung des subsidiären Schutzstatus (nicht auf die Zuerkennung des Flüchtlingsstatus) und auf die Beendigung sowohl des Flüchtlings- als auch des subsidiären Status. Die blosse Einschränkung der Statusrechte ist hingegen nicht von der Vermischung betroffen.

Grund für die Vermischung bezüglich der Nichtzuerkennung des subsidiären Schutzstatus ist, dass sich die Richtlinie dabei aus zwei ganz verschiedenen Quellen der Flüchtlingskonvention bedient. Einerseits aus Art. 1 F GFK, der die Gründe für den Ausschluss aus der Flüchtlingseigenschaft festlegt, andererseits aus Art. 33 Abs. 2 GFK, der festlegt, unter welchen Umständen Flüchtlinge vom Schutz des (flüchtlingsrechtlichen) Refoulement ausgenommen sind. Während die GFK in Art. $1 \mathrm{~F}$ die doppelte Zielsetzung verfolgt, schutzunwürdige Personen aus einem Schutzstatus auszuschliessen und zu verhindern, dass sich eine Person der Strafverfolgung entzieht, sieht die GFK für Sicherheitsbedenken im Aufnahmestaat andere Mechanismen vor, wie die Unterordnung dieser Person unter das nationale Strafrecht (S. 224). In Art. 17 Abs. 1 lit. d der Richtlinie wird diese dritte Zielsetzung aber noch hinzugefügt.

9 Das führt zu dem paradoxen Ergebnis, dass sich die Gewährung des subsidiären Schutzstatus zwar an der Rechtsprechung des EGMR zum menschenrechtlichen Refoulement-Verbot orientiert, sich die Gründe für die Nichtgewährung gerade über diese Rechtsprechung hinwegsetzen und einen Ausschluss aus einem Status vorsehen, wo eine Rückführung völkerrechtlich verboten bleibt (S. 219). Hinzu kommt noch, dass die Mitgliedstaaten die Ausschlussgründe zusätzlich selber erweitern können (Art. 17 Abs. 3 RL 2011/95).

10 Ein ähnliches Durcheinandergeraten von Konzepten ortet Egbuna-Joss auch in der Bestimmung, welche die Beendigung, Aberkennung und Ablehnung der Verlängerung des Flüchtlingsstatus regelt (Art. 14 RL 2011/95). Schon der Titel der Bestimmung offenbart das Problem, indem er Erlöschens- und Ausweisungsgründe vermengt (S. 253). Hier ist es wiederum die Nachbildung der Gründe für den Ausschluss aus der Flüchtlingseigenschaft aus Art. 1 F GFK und der Gründe für die Ausweisung einer Person in Art. 32 und 33 Abs. 2 GFK, die zu der Vermischung führt. Während in der Konzeption der Konvention die ersteren Gründe zum Ausschluss aus dem Status des Flüchtlings führen und die zweiten zur Wegweisung aus einem Land unter Beibehaltung des Status des Flüchtlings, führen nach der Richtlinie beide Tatbe- 
stände zum Verlust des Status, den die Richtlinie vorsieht.

\section{Aber wie gross ist das Problem?}

11 Während der Nachweis dieser Kategorienvermischung klar gelingt, erschliesst sich weniger unmittelbar, wie problematisch sie ist. Als eindeutig völkerrechtswidrig identifiziert Egbuna-Joss lediglich die faktische Schaffung von Ausschlussgründen, die in der Konvention nicht vorgesehen sind. Der Verstoss ist insofern flagrant, als die Richtlinie selber explizit sagt, die Rechtsstellung könne einem «Flüchtling» vorenthalten werden, sich also im Klaren ist, dass die Personen, die aus den Statusrechten ausgeschlossen werden sollen, Flüchtlinge im Rechtssinne sind (Art. 14 Abs. 4 und 5 RL 2011/95). Es handelt sich hierbei allerdings um Kann-Vorschriften.

Bezüglich der Bestimmungen für die Nichtgewährung und die Beendigung des subsidiären Status muss in Betracht gezogen werden, dass dieser erst durch die Richtlinie geschaffen worden ist. Wo sich die Vermischung der völkerrechtlichen Kategorien also nur auf den subsidiären Status bezieht, ist ein Ausschluss aus diesem völkerrechtlich ohne weiteres zulässig, solange das völkerrechtliche Minimum des menschenrechtlichen Refoulement-Verbotes eingehalten wird. Dasselbe gilt auch für die Beendigung dieses Status, wie die Autorin festhält (S. 264).

Es bleibt daher zunächst unklar, warum diese Vermischung mehr ist als eine rechtssystematische Unschönheit. Die Autorin befürchtet, die Vermischung sei ein Resultat des Lobby-Efforts jener Mitgliedstaaten, die schon lange eine Auf- weichung der Refoulement-Praxis des EGMR forderten. Mit der Qualifikationsrichtlinie hätten diese nun einen neuen Hebel gegen den EGMR. Die Vermischung der Kategorien könnte ausserdem über Europa hinaus ausstrahlen, an Orte, wo nicht der EGMR als letzter Garant zur Verfügung steht (S. 220).

14 Angesichts der starken Reaktionen, welche die hier aufgegriffenen Probleme der Qualifikationsrichtlinie bei internationalen Organisationen hervorgerufen haben, scheint es hingegen eher wahrscheinlich, dass sich der EGMR seiner Rolle als letztes Sicherheitsnetz umso stärker bewusst ist und dass internationale Organisationen die Wichtigkeit des menschenrechtlichen Refoulement-Verbotes zusätzlich hervorheben werden.

15 Egbuna-Joss weist darauf hin, dass durch die Vermischung von Kategorien das Harmonisierungsziel verfehlt wurde, mit dem die Qualifikationsrichtlinie eigentlich angetreten war. Sie führt zwar einen subsidiären Schutzstatus ein, um dem menschenrechtlichen RefoulementVerbot Rechnung tragen zu können, ermöglicht einen Ausschluss aus diesem Status dann aber auch in einigen jener Fälle wieder, in denen das menschenrechtliche Refoulement-Verbot nach wie vor greift. Auch das mag ein Ärgernis sein. Es ändert aber nichts daran, dass die Einführung eines subsidiären Schutzstatus ein Fortschritt war und die Fälle, in denen Menschen zwar nicht zurückgeschickt werden dürfen aber ohne Status bleiben, im Vergleich zu früher verhältnismässig selten sind, weshalb das Harmonisierungsziel jedenfalls zum Teil erreicht worden ist. 
Eine möglicherweise völkerrechtswidrige Situation ortet die Autorin in der Kombination mit der Verfahrensrichtlinie (RL 2013/32), die in ihrem Art. 32 (unter Verweis auf die Qualifikationsrichtlinie) den Mitgliedstaaten freistellt, die Ausnahmetatbestände zum flüchtlingsrechtlichen Refoulement-Verbot schon vor der Überprüfung der Flüchtlingseigenschaft zu prüfen. Diese festgestellte Völkerrechtswidrigkeit setzt allerdings die Annahme voraus, die Richtlinie stelle es den Mitgliedstaaten frei, von den Vorgaben der Konvention abzuweichen (S. 267).

Dagegen lässt sich natürlich einwenden, sowohl aus der Richtlinie selber (Erwägung 3 und 4) als auch aus dem Grundsatz der Völkerrechtsfreundlichkeit der Unionsrechtsordnung und deren Bindung an die Menschenrechte (welche für die gemeinsame Asylpolitik in Art. 78 Abs. 1 AEUV noch einmal explizit bekräftigt wird) ergebe sich, dass es den Mitgliedstaaten nach wie vor nicht frei steht, die Konvention zu verletzen. Die Autorin gelangt an anderer Stelle selber auch zu diesem Schluss (S. 122).

\section{Wie konnte das passieren?}

18

Das Problem der Vermischung von Kategorien durch die Richtlinie ist recht offensichtlich. Es wurde bereits in Bezug auf die Vorgängerrichtlinie und den Entwurf der überarbeiteten Richtlinie durch das UNHCR und zahlreiche NGOs bemängelt.2 Es war der Kommission, dem

2 Statt vieler: UNHCR comments on the European Commission's proposal for a Directive of the European Parliament and of the Council on minimum standards for the qualification and status of third country nationals or stateless persons as beneficiaries of international protection and the
Rat und dem Parlament also offensichtlich bewusst und wurde dennoch in Kauf genommen. ${ }^{3}$ Dafür muss es eine Erklärung geben. Die Überlegungen dazu müssen eine Spur hinterlassen haben. War eine gezielte Strategie der Mitgliedstaaten im Spiel, die Ausschlussgründe über diejenigen, der Konvention hinaus auszudehnen, 4 oder eine bewusste $\mathrm{Zu}$ rückweisung der Haltung des UNHCR, dass die Zuerkennung des Flüchtlingsstatus deklaratorisch und nicht konstitutiv sei?5

19 Für die Beurteilung der Völkerrechtskonformität der Richtlinie wäre diese Erklärung von grossem Interesse. Man mag es daher bedauern, dass weder der zweite Teil, der sich mit der Genese des gemeinsamen europäischen Asylsystems befasst, noch der dritte Teil, der die Qualifikationsrichtlinie auf ihre Völkerrechtskonformität untersucht, auf die Materialien zur Entstehung der Richtlinie zurückgreift, die dazu vertieft hätten Auskunft geben können.

20 Wenig thematisiert bleibt im Übrigen, inwiefern der Ausschluss aus einem Schutzstatus überhaupt je einen sinnvollen Beitrag zur Sicherheit eines Staates leisten kann, solange die Person dennoch auf Grund des menschenrechtlichen Refoulement-Verbotes in diesem Staat bleiben kann. Ein möglicher Grund dafür,

content of the protection granted (COM(2009) 551, 21 October 2009) vom Juli 2010, S. 13 f.

3 Siehe etwa ECRE Information Note on the Directive 2011/95/EU of the European Parliament and of the Council of 13 December 2011, S. $10 \mathrm{f}$.

4 Vgl. Hierzu Comments of the ECRE on the European Commission Proposal to recast the Qualification Directive vom März 2010.

5 Asylum in the European Union - A Study of the Implementation of the Qualification Directive, UNHCR, November 2007, S. 90 ff., 94. 
dass diese Diskussion unterbleibt, ist, dass der Begriff der öffentlichen Sicherheit und Ordnung relativ grob umrissen bleibt.

\section{Zum Begriff der Sicherheit und Ordnung}

21 Der Begriff der Sicherheit und Ordnung ist auslegungs- und konkretisierungsbedürftig (vgl. S. 153). Geht es um militärische Sicherheit, um die Beziehung zu anderen Staaten, um den Schutz vor Kriminalität (wenn ja, vor welcher?), um die Verhinderung von Terrorismus, um den Schutz des Asylsystems vor «Missbrauch» oder vor schutzunwürdigen Personen, um die Durchsetzbarkeit von Wegweisungen oder um eine Mischung aus all diesen Zielsetzungen? EgbunaJoss erklärt die stärkere Betonung von Sicherheit als Rechtsgut und politisches Ziel im Flüchtlingswesen in erster Linie mit dem Ereignis des 11. September 2001 (S. 9). Doch in welchem Verhältnis steht dieses Ereignis zu den Bestrebungen, Justizflüchtlinge zu verhindern, die bereits in der Internationalen Flüchtlingsorganisation (1946-1951) angelegt sind (S. 17)? In welchem Verhältnis stehen die Idee der Schutzunwürdigkeit gewisser verfolgter Personen und das Anliegen, die öffentliche Sicherheit zu schützen? Wie stehen das Bestreben, «Missbrauch» $\mathrm{zu}$ verhindern und das Rechtsgut «Glaubwürdigkeit des Schutzregimes» (vgl. Anhang) und das Bestreben um Sicherheit zueinander?

Viele dieser Bezüge erschliessen sich nicht vollständig, auch wenn der Begriff der nationalen Sicherheit und der öffentlichen Ordnung, wie ihn die Qualifikationsrichtlinie verwendet, etwas umrissen wird und insbesondere klar wird, dass die Richtlinie eine gegenwärtige und erhebliche Gefahr verlangt, die von einer schutzsuchenden Person ausgeht (S. 156). Insbesondere das Verhältnis von Sicherheit und Schutzunwürdigkeit bleibt aber auch in den Schlussfolgerungen ambivalent (S. 273). Die Autorin weist selber darauf hin, dass es bei der Figur der Schutzunwürdigkeit nicht in erster Linie um die Gefahrenabwehr gehe.

23 Es bleibt die Frage, ob nicht der Import des Konzepts der Versicherheitlichung (Securitization) aus den Politikwissenschaften sinnvolle Beiträge hätte leisten können, um zu konkretisieren, wie sich das Bedürfnis nach Sicherheit über die Zeit veränderte und in der Richtlinie Niederschlag fand. Besonders die auffällig starke Verortung in den Ereignissen des 11. Septembers 2001 hätte an Plausibilität gewonnen, wenn der Wandel unter Rückgriff auf sozialwissenschaftliche Theorien erklärt worden wäre. Auch für den Vergleich von regionalen Schutzinstrumenten im ersten Teil der Arbeit und der Art, wie sie dem Bedürfnis nach Sicherheit und Ordnung Rechnung tragen, mag man es bedauern, dass der Begriff nicht stärker theoretisch eingebettet worden ist. Für das Verständnis von Unterschieden regionaler Sicherheitskonzepte wäre das von grossem Interesse gewesen.

24 Insgesamt bleibt die Arbeit aber nicht nur ein wertvoller Beitrag als Kommentar der einschlägigen Artikel der Richtlinien und als Einführung in deren Systematik, sie ist auch ein nützliches und praktisches Nachschlagwerk. Andererseits macht die Arbeit neugierig auf ver- 
tiefte Einblicke in die praktischen Konsequenzen der Kategorienvermischung, die sie feststellt, auf eine Aufarbeitung der Ursprünge in den Materialien und auf ihre Einbettung in einen Dialog mit den Sozialwissenschaften, die sich ihrerseits mit der Versicherheitlichung des Asylwesens beschäftigen.

«Der Schutz der öffentlichen Ordnung
und Sicherheit im Rahmen der internati-
onalen Schutzgewährung - Eine Analyse
der Qualifikationsrichtlinie 2011/95 der
Europäischen Union unter besonderer
Berücksichtigung der völkerrechtlichen
Vorgaben», Andrea Egbuna-Joss, Carl
Grossmann Verlag, Berlin/Bern 2016,
ISBN: 978-3-941159-O4-4 (gedruckte
Ausgabe, Hardcover), ISBN: 978-3-
941159-05-1 (e-Book, Open Access)

